\title{
Relationship of sodium intake with obesity among Korean children and adolescents: Korea National Health and Nutrition Examination Survey
}

\author{
Soo-Kyung Lee ${ }^{1}$ and Mi Kyung Kim ${ }^{2 *}$ \\ ${ }^{1}$ Department of Food and Nutrition, Inha University, Incheon 402-751, Korea \\ ${ }^{2}$ National Cancer Center, Division of Cancer Epidemiology and Management, Goyang, Gyeonggi 411-769, Korea \\ (Submitted 23 June 2015 - Final revision received 24 November 2015 - Accepted 30 November 2015 - First published online 13 January 2016)
}

\section{Abstract}

We investigated whether dietary and urinary $\mathrm{Na}$ is associated with adiposity in Korean children and adolescents (10-18 years), a population with a high salt intake. Study subjects were Korean children and adolescents who participated in the cross-sectional nationally representative Korea National Health and Nutrition Examination Survey (2010-2011). This study used measures of dietary (24-h dietary recall) and urinary $\mathrm{Na}$ (Na:creatinine ratio) and three methods to determine obesity (BMI, waist circumference (WC) and total body per cent fat (TBPF)). Higher $\mathrm{Na}$ intake was significantly associated with obesity, adjusting for the covariates. Subjects in the highest tertile of urinary Na excretion had a significantly higher OR for higher adiposity compared with those in the lowest tertile (multivariate-adjusted OR $3 \cdot 13$ (95\% CI $1 \cdot 81$, $5 \cdot 50$ ) for BMI, 2.15 (95\% CI 1.27, 3.66) for WC and 1.92 (95\% CI 1.29, 2.86) for TBPF, respectively). Na intake estimated by the $24-\mathrm{h}$ recall method also showed significant association with adiposity (multivariate-adjusted OR 2.79 (95\% CI 1.66, 4.68) for BMI and 2.14 (95\% CI 1.25, 3.67) for WC, respectively). The significant associations between $\mathrm{Na}$ and adiposity remained significant after additionally adjusting for sugar-sweetened beverage (SSB) consumption. Our results revealed a significant positive association between urinary and dietary Na and adiposity in Korean children and adolescents, independent of SSB consumption.

Key words: Sodium: Obesity: Children: Korea National Health and Nutrition Examination Surveys

High $\mathrm{Na}$ intake has been linked to a variety of increased health risks such as hypertension ${ }^{(1,2)}, \mathrm{CVD}^{(3,4)}$, stomach cancer $^{(5-7)}$ and type 2 diabetes ${ }^{(8)}$. In addition to the traditionally recognised health effects of high $\mathrm{Na}$ intake, obesity has recently gained much interest as another possible related health outcome (5) $^{\text {. }}$

Although several studies including a longitudinal study have reported significant associations between $\mathrm{Na}$ intake and obesity among adults ${ }^{(9-14)}$, relatively limited evidence is available among children and adolescents ${ }^{(15-18)}$. Investigation of the associations between $\mathrm{Na}$ intake and obesity, however, has been limited by weakness of assessment methods of both $\mathrm{Na}$ intake and obesity. Quantification of dietary salt intake is imperfect because of the limitations in dietary assessment methods and food databases ${ }^{(19,20)}$. The 24-h urinary $\mathrm{Na}$ excretion measurement, generally considered as the gold standard ${ }^{(20)}$, is also limited by the difficulty of urine collection for $24 \mathrm{~h}$. Spot urine or half-day urine measurements have been proposed as substitutes for the 24-h urine measurement in an effort to overcome the difficulty ${ }^{(21-23)}$. For obesity measures, most studies, except one study ${ }^{(13)}$, used either BMI or waist circumference (WC). Although both BMI and WC are valid and widely used measures, they have their own limitations such as not being able to control for muscle mass or total fat mass ${ }^{(24)}$.

Although mechanisms of the association between $\mathrm{Na}$ intake and obesity have not been well established, the role of soft drink consumption has been explored and shown to be significant among children and adolescents in the Western food culture $^{(25)}$. This hypothesis indicates that higher energy intake from sugar-sweetened beverages (SSB) consumed to quench thirst caused by high $\mathrm{Na}$ intake would contribute to obesity $^{(26,27)}$. Whether this pathway would also be important in non-Western food cultures has not been examined, however, Koreans are reported to have approximately three times more salt intake than the recommended level, affording a large variation of exposure level ${ }^{(28,29)}$. Korean food culture is strongly preserved with wide-range influences of Western food culture, which makes it an interesting population to investigate for an association between $\mathrm{Na}$ and obesity.

Therefore, this study examined whether urinary and dietary $\mathrm{Na}$ exposure showed independent associations with obesity across multiple obesity measures - obesity measured by BMI, abdominal obesity measured by WC and per cent body fat measured by dual-energy X-ray absorptiometry (DEXA) - in a

Abbreviations: DEXA, dual-energy X-ray absorptiometry; KNHANES, Korea National Health and Nutrition Examination Survey; MET, metabolic equivalents; SSB, sugar-sweetened beverage; TBPF, total body per cent fat; WC, waist circumference.

* Corresponding author: M. K. Kim, fax +82 31920 2006, email alrud@ncc.re.kr 
nationally representative sample of Korean children and adolescents aged from 10 to 18 years.

\section{Methods \\ Study design}

This study analysed the Korea National Health and Nutrition Examination Survey (KNHANES). The KNHANES is an annually conducted and nationally representative survey, consisting of a health interview, behaviour and nutrition surveys and a medical examination. This cross-sectional survey utilised a stratified, multistage, cluster probability sampling design with the National Census Registry. More details of the survey design and methods are available elsewhere ${ }^{(30)}$. This study chose to use data from 2010 to 2011 because urinary $\mathrm{Na}$ excretion data were collected only during these 2 years ${ }^{(31)}$

The KNHANES is conducted in accordance with the Helsinki Declaration. Written informed consent was obtained from parents or guardians. Data without personal identifiable information were provided for this analysis.

\section{Study participants}

Among children aged between 10 and 18 years ( $n$ 1873), whose body weight, height and WC were recorded in the KNHANES 2010-2011, we excluded children with missing diet information ( $n$ 195) and extreme energy intake ( $n$ 12); the final analytical sample consisted of 1467 children and adolescents. Extreme energy intake was defined as $<1673 \mathrm{~kJ} / \mathrm{d}(400 \mathrm{kcal} / \mathrm{d}$ ) or $>23$ $848 \mathrm{~kJ} / \mathrm{d}(5700 \mathrm{kcal} / \mathrm{d})$ for 10-11-year-old boys, $<2510 \mathrm{~kJ} / \mathrm{d}$ $(600 \mathrm{kcal} / \mathrm{d})$ or $>33890 \mathrm{~kJ} / \mathrm{d}(8100 \mathrm{kcal} / \mathrm{d})$ for $12-18$-year-old boys, $<1569 \mathrm{~kJ} / \mathrm{d}(375 \mathrm{kcal} / \mathrm{d})$ or $>21338 \mathrm{~kJ} / \mathrm{d}(5100 \mathrm{kcal} / \mathrm{d})$ for 10-11-year-old girls and $<2092 \mathrm{~kJ} / \mathrm{d} \quad(500 \mathrm{kcal} / \mathrm{d})$ or $>25104 \mathrm{~kJ} / \mathrm{d}(6000 \mathrm{kcal} / \mathrm{d})$ for 12-18-year-old girls, respectively $^{(32)}$. For the analysis of per cent body fat measured by DEXA, we only included subjects ( $n$ 1019) whose DEXA measurements were available. The age of 10 years had to be used as the lowest age because urine samples were available of individuals aged 10 years or above.

\section{Measures}

KNHANES utilises mobile examination centres where health interviews and medical examinations are conducted. The nutrition survey was conducted approximately 1 week after the health examination. This study used socio-demographic and health behaviour information from the health interviews, urinary $\mathrm{Na}$, body fat and anthropometric information from the medical examination and dietary information from the nutrition survey.

Sodium intake. Na intake was assessed using two different methods: (1) dietary Na intake per $4184 \mathrm{~kJ}$ (1000 kcal) from a 24-h recall and (2) urinary Na:creatinine ratio. Dietary Na intake was calculated from 24-h recalls conducted by trained nutritionists with tools to help portion size estimation. To control for the reported tendency that higher food intake is naturally associated with higher $\mathrm{Na}$ intake ${ }^{(33)}$, dietary $\mathrm{Na}$ density (dietary $\mathrm{Na}$ intake $(\mathrm{mg}) / 4184 \mathrm{~kJ}(1000 \mathrm{kcal}))$ was calculated and used in the analyses. The KNHANES provided urinary $\mathrm{Na}$ and creatinine values from the overnight spot urine samples. From these values, we calculated the urinary $\mathrm{Na}$ :creatinine ratio.

Adiposity was also assessed by measuring three different parameters: (1) BMI, (2) WC and (3) total body per cent fat (TBPF). BMI was selected because it is one of the most widely used and recommended methods to determine the obesity status of children and adolescents ${ }^{(34)}$. Height and weight were measured in the mobile examination centre following standardised measurement protocols. BMI was calculated by dividing body weight by the squared body height. The age- and sexadjusted BMI $Z$ score was obtained using a Korean growth chart $^{(35)}$. Children and adolescents were considered to be obese if their BMI was of the 95th percentile or greater for their age and sex or if their BMI was $25 \mathrm{~kg} / \mathrm{m}^{2}$ or higher, and overweight was defined as having 85 th $\leq \mathrm{BMI}<95$ th percentile for age and sex ${ }^{(35,36)}$. This study used WC as a measure of abdominal adiposity $^{(37)}$. WC was measured at the narrowest area between the lowest rib and the uppermost lateral border of the right iliac crest, and a WC of the 90th percentile for age and sex or greater was used as a cut-off value for abdominal adiposity ${ }^{(35,38,39)}$. TBPF was obtained by DEXA using a QDR Discovery fan beam densitometer (Hologic Inc.) according to the recommended procedures from the manufacturer. DEXA examinations were included as a part of the medical examination in 2010 and 2011; therefore, analyses of TBPF were carried out in a smaller sample ( $n$ 1019). The DEXA method proved to be a valid assessment tool to measure body fat ${ }^{(40)}$. Adiposity was also defined as having a TPBF $>25 \%$ for boys, $>30 \%$ for girls younger than 11 years and $>35 \%$ for girls aged 11 years or older ${ }^{(41)}$.

Sugar-sweetened beverages. SSB were obtained using a single 24-h recall questionnaire, and included energetic sweeteners such as sugar-sweetened soda, soft drinks, fruit drinks, sport drinks, energy drinks, sugar-sweetened tea or sweetened milk $^{(42)}$. The KNHANES food-group classification system was used to identify food groups that fell within the definition of SSB. These food codes were used to aggregate data to calculate the SSB $(\mathrm{g} / \mathrm{d})$.

Adjusting variables. Adjusting variables included age, sex and household income obtained from the Health Interview and Behavior Surveys, which were obtained through face-to-face interviews by trained interviewers. Household income quartile was used in the analyses. Physical activity was assessed by a pre-validated questionnaire in the Health Interview and Behavior Survey. The subjects specified how many hours of a day they spent doing vigorous, moderate and light activities for a usual 1 week. Subsequently, metabolic equivalents (MET)-h/ week was estimated for each subject by multiplying together the number of hours per week a woman spent in a particular activity and the estimated MET score for that activity. Total energy intake was calculated using the $24-\mathrm{h}$ recall data from the Nutrition Survey.

\section{Statistical analysis}

All the analyses were carried out using the survey procedures provided in SAS software 9.2, accounting for the complex 
survey design used, and following the analytical guidelines for KNHANES data from the Korea Center for Disease Control and Prevention $^{(30)}$ to estimate nationally representative values and to extrapolate the findings to the entire Korean population. Distributions of general characteristics are presented as mean values with their standard errors or weighted percentages. Differences between groups were analysed using survey means or weighted percentages for survey design. We checked the normality of urinary and dietary $\mathrm{Na}$ variables, which were not normally distributed. These exposure variables were then log-transformed, and we carried out further analysis. In addition, logistic regression analyses were carried out for tertiles of $\mathrm{Na}$ variables. A survey logistic regression was used to test the associations between tertile of $\mathrm{Na}$ measurements and adiposity and to calculate the crude and multivariate-adjusted OR along with $95 \%$ CI for each tertile compared with the lowest tertile. Two multivariate models were tested to explore the roles of $\mathrm{Na}$ in relation to adiposity. Model 1 adjusted for age, sex (only for analysis of sex combined), household income, total daily energy intake (only for urinary $\mathrm{Na}$ variables) and physical activity (MET). To examine the effect of soft drink consumption, model 2 additionally adjusted for soft drink consumption in addition to all the mentioned variables in model 1 . Separate analyses for each sex were also carried out. The $P$ values used to test for linear trends were calculated using $\mathrm{Na}$ measurements as continuous variables after adjustment for confounding factors. The significance level of 0.05 was used for all statistical tests.

\section{Results \\ Characteristics of the participants}

Basic characteristics of socio-demographic factors, Na intake, adiposity, soft drink consumption and adjusting variables are presented in Table 1 . The participants were 1467 children and adolescents with an average age of 13.4 years, and $43.6 \%$ of the participants were girls. The daily $\mathrm{Na}$ intake assessed by 24-h recalls was $4305 \mathrm{mg}$. The average $\mathrm{Na}$ excretion estimated by morning spot urine analysis was $138 \mathrm{mg}$. Boys excreted $\mathrm{Na}$ significantly more than girls $(P=0.0007)$, and the difference remained significant after controlling for muscle mass by dividing the $\mathrm{Na}$ value with creatinine. The children showed an average BMI of $20.7 \mathrm{~kg} / \mathrm{m}^{2}$ and a WC of $69.5 \mathrm{~cm}$. The average TBPF was $26 \cdot 6 \%$. Although average values of WC were significantly higher in boys than in girls $(P<0.0001)$, adiposity rate by WC was higher in girls than in boys $(P=0.0254)$. Approximately $59 \%$ of the participants reported soft drink consumption, and soft drink consumers drank on average $398 \mathrm{~g} / \mathrm{d}$ of soft drinks. Boys showed higher soft drink

Table 1. Characteristics of the participants

(Mean values with their standard errors; percentages)

\begin{tabular}{|c|c|c|c|c|c|c|c|}
\hline \multirow[b]{2}{*}{ Variables } & \multicolumn{2}{|c|}{ Total ( $n$ 1467) } & \multicolumn{2}{|c|}{ Boys ( $n$ 827) } & \multicolumn{2}{|c|}{ Girls ( $n$ 640) } & \multirow[b]{2}{*}{$P^{*}$} \\
\hline & Mean & SE & Mean & SE & Mean & SE & \\
\hline \multicolumn{8}{|l|}{ Predictor variables } \\
\hline Dietary $\mathrm{Na}$ intake from $24-\mathrm{h}$ recall $(\mathrm{mg} / \mathrm{d})$ & 4305 & $104 \dagger$ & 4799 & 138 & 3640 & 112 & $<0.0001$ \\
\hline Dietary Na density $(\mathrm{mg} / 4184 \mathrm{~kJ}$ per $\mathrm{d}(1000 \mathrm{kcal}$ per $\mathrm{d}))$ & 1961 & 33.9 & 1976 & 41.8 & 1940 & 47.9 & 0.5136 \\
\hline Spot urinary $\mathrm{Na}$ excretion $(\mathrm{mmol} / \mathrm{l})$ & 138 & 1.98 & 143 & $2 \cdot 68$ & 130 & 2.74 & 0.0007 \\
\hline Spot urinary Na:creatinine ratio & 8.97 & 0.20 & 9.33 & 0.28 & 8.49 & 0.26 & 0.0350 \\
\hline \multicolumn{8}{|l|}{ Outcome variables } \\
\hline $\mathrm{BMI}\left(\mathrm{kg} / \mathrm{m}^{2}\right)$ & 20.7 & 0.12 & 20.9 & 0.16 & $20 \cdot 4$ & 0.21 & 0.0689 \\
\hline Overweight by BMI (\%)† & \multicolumn{2}{|c|}{$6 \cdot 70$} & \multicolumn{2}{|c|}{5.44} & \multicolumn{2}{|c|}{8.39} & 0.0498 \\
\hline Obesity by BMI (\%)† & \multicolumn{2}{|c|}{$12 \cdot 2$} & \multicolumn{2}{|c|}{$13 \cdot 2$} & \multicolumn{2}{|c|}{$10 \cdot 8$} & 0.3433 \\
\hline WC $(\mathrm{cm})$ & 69.5 & 0.34 & 71.2 & 0.44 & $67 \cdot 3$ & 0.54 & $<0.0001$ \\
\hline Adiposity by WC (\%)† & \multicolumn{2}{|c|}{9.81} & \multicolumn{2}{|c|}{7.89} & \multicolumn{2}{|c|}{$12 \cdot 4$} & 0.0254 \\
\hline TBPF (\%) & $26 \cdot 6$ & 0.37 & 23.0 & 0.44 & $32 \cdot 0$ & 0.41 & $<0.0001$ \\
\hline Adiposity by TBPF (\%)†‡ & \multicolumn{2}{|c|}{$35 \cdot 2$} & \multicolumn{2}{|c|}{37.4} & \multicolumn{2}{|c|}{$32 \cdot 2$} & 0.1843 \\
\hline \multicolumn{8}{|l|}{ Adjusting variables } \\
\hline Age (years) & $13 \cdot 4$ & 2.53 & $13 \cdot 5$ & $2 \cdot 49$ & $13 \cdot 4$ & 2.57 & 0.1435 \\
\hline Household income (\%) & \multirow{2}{*}{\multicolumn{2}{|c|}{$16 \cdot 1$}} & & & \multirow{2}{*}{\multicolumn{2}{|c|}{$16 \cdot 6$}} & 0.2143 \\
\hline$<\$ 650$ & & & \multicolumn{2}{|c|}{$15 \cdot 8$} & & & \\
\hline$\$ 650-\$ 1265$ & \multicolumn{2}{|c|}{$30 \cdot 6$} & \multicolumn{2}{|c|}{$28 \cdot 2$} & \multicolumn{2}{|c|}{33.8} & \\
\hline$\$ 1265-\$ 2047$ & \multicolumn{2}{|c|}{$27 \cdot 8$} & \multicolumn{2}{|c|}{28.5} & \multirow{2}{*}{\multicolumn{2}{|c|}{$\begin{array}{l}26.8 \\
22.8\end{array}$}} & \\
\hline$\geq \$ 2047$ & \multicolumn{2}{|c|}{$25 \cdot 5$} & \multicolumn{2}{|c|}{27.5} & & & \\
\hline Total energy intake $(\mathrm{kJ} / \mathrm{d})$ & 9242 & 137.6 & 10263 & $199 \cdot 1$ & 7870 & $137 \cdot 6$ & $<0.0001$ \\
\hline Total energy intake $(\mathrm{kcal} / \mathrm{d})$ & 2209 & 32.9 & 2453 & 47.6 & 1881 & 32.9 & \\
\hline SSB consumer (weighted \%) & & & & & & & 0.5353 \\
\hline SSB per consumer $(\mathrm{g} / \mathrm{d}) \S$ & 398 & $18 \cdot 2$ & 437 & 27.5 & 347 & $18 \cdot 2$ & 0.0059 \\
\hline SSB per capita $(\mathrm{g} / \mathrm{d})$ & 235 & $13 \cdot 7$ & 254 & $20 \cdot 0$ & 209 & $14 \cdot 2$ & 0.0468 \\
\hline MET $(\mathrm{h} / \mathrm{d})$ & 31.4 & 1.69 & 37.2 & 2.58 & 23.6 & 1.90 & $<0.0001$ \\
\hline
\end{tabular}

WC, waist circumference; TBPF, total body per cent fat; SSB, sugar-sweetened beverages; MET, metabolic equivalents.

* Categorical variables were analysed using the $x^{2}$ test; continuing variables were analysed using the $t$ test.

$\dagger$ Obesity was defined as having a BMI $\geq 95$ th percentile for age and sex or $\geq 25 \mathrm{~kg} / \mathrm{m}^{2}$; overweight was defined as having 85 th $\leq \mathrm{BMI}<95$ th percentile for age and sex; abdominal adiposity as a WC $\geq 90$ th percentile for age and sex in the study sample; as a TPBF $>25 \%$ for boys, $>30 \%$ for girls younger than 11 years and $>35 \%$ for girls aged 11 years or older. $\ddagger$ Analysis was conducted $(n 1019)$ in a subsample of the subjects with dual-energy X-ray absorptiometry, the TBPF.

$\S$ Analysis was completed in a subsample of SSB consumers only. 
consumption than girls $(P=0.0059)$. The average MET was $31.4 \mathrm{~h} / \mathrm{d}$, and boys reported significantly higher MET than girls $(P<0 \cdot 0001)$.

\section{Association between urinary sodium excretion and adiposity}

The spot urinary Na:creatinine ratio (Table 2), used to estimate dietary $\mathrm{Na}$ intake, showed significant associations with BMI, WC and TBPF $(P<0.05)$. Using BMI, risk of 'overweight' was not significantly associated with urinary $\mathrm{Na}$ :creatinine ratio, but risk of 'obesity' showed consistent and significant association with higher multivariate OR around three than OR of WC or TBPF. Significant dose-response relationships were also found in associations between urinary $\mathrm{Na}$ :creatinine ratio and risk of obesity assessed by BMI in both boys and girls $(P<0.05)$.

The urinary Na:creatinine ratio showed significant association with risk of adiposity assessed by WC only in boys in the highest tertile group with OR of 2.26 (95\% CI 1.02, 4.99). The dose-response relationship was also significant at $P<0.05$. The multivariate-adjusted OR for adiposity risk assessed by TBPF in children of the highest tertile of urinary Na:creatinine ratio in model 1, compared with the lowest tertile, were $2 \cdot 13$ (95\% CI $1.15,3.97$; $\left.P_{\text {for trend }}=0.0166\right)$ for boys and 1.75 (95\% CI 0.96 , 3.24; $P_{\text {for trend }}=0.0662$ ) for girls, respectively.

The addition of soft drink consumption into the multivariate logistic model (model 2) did not create any statistically significant differences in the patterns of the association between urinary $\mathrm{Na}$ excretion and adiposity (Table 2). Soft drink consumption itself was not significantly associated with the risk of adiposity (data not shown).

\section{Association between dietary sodium intake and adiposity}

Table 3 shows varying degrees of associations between dietary $\mathrm{Na}$ density $(\mathrm{Na} / 4184 \mathrm{~kJ}(1000 \mathrm{Kcal})$ of E) estimated from 24-h recalls and the three adiposity measures. The multivariateadjusted OR for overweight risk assessed by BMI, obesity risk assessed by BMI and adiposity risk assessed by WC in children of the highest tertile of dietary Na density in model 1, compared with the lowest tertile, were $2 \cdot 11$ (95\% CI 1.14, 3.90), 2.73 (95\% CI $1.65,4.51)$ and 1.95 (95\% CI 1.17, 3.26), respectively. Significant dose-response relationships were found with overweight risk assessed by BMI, adiposity risk assessed by BMI and adiposity risk assessed by WC $\left(P_{\text {for trend }}=0 \cdot 0207,<0 \cdot 001\right.$ and 0.0086, respectively). Although girls showed a significant association of dietary $\mathrm{Na}$ intake with overweight risk assessed by BMI and obesity risk assessed by BMI $(P<0.05)$, boys reported a significant association with obesity risk assessed by BMI. Significant associations between adiposity risk assessed by WC and dietary $\mathrm{Na}$ intake were only found in the total sample, but no consistently significant associations were found for each sex. We found no significant associations between adiposity risk assessed by TBPF and dietary $\mathrm{Na}$ intake.

Adding soft drink consumption to the model (model 2) did not alter the general pattern of the associations described above (model 1). Overall, the association between BMI-obesity and dietary $\mathrm{Na}$ density appeared to be the strongest in terms of size of OR and consistency of significant dose-response relationships in both models 1 and 2 (Table 3 ).

\section{Discussion}

This study found that higher $\mathrm{Na}$ intake was significantly associated with adiposity in children and adolescents, after adjusting for energy intake, SSB intake, physical activity and socio-demographic variables. The results showed generally consistent patterns of association regardless of the assessment methods of $\mathrm{Na}$ intake or measure of adiposity. Risk of obesity assessed by BMI was consistently and significantly associated with urinary $\mathrm{Na}$ :creatinine ratio and dietary $\mathrm{Na}$ intake, whereas risk of overweight assessed by BMI, risk of abdominal adiposity assessed by WC and TBPF showed relatively weaker and less consistent association with $\mathrm{Na}$ intake. Along with consistently significant dose-response relationships between $\mathrm{Na}$ intake and obesity, findings of this study suggest that higher $\mathrm{Na}$ intake was related to obesity and abdominal adiposity to a lesser degree.

This study also explored a possible role of SSB consumption, using soft drink consumption as a proxy variable, in the association between $\mathrm{Na}$ intake and obesity in Korean children and adolescents. The addition of soft drink consumption into the multivariate logistic regression models did not make meaningful differences across assessment methods and sex, which means there appears to be $\mathrm{Na}$ intake effects independent of soft drink consumption in this population. This finding is not in agreement with previously reported findings from the US population $^{(26)}$ and the Australian population ${ }^{(27)}$. Approximately $59 \%$ of Korean children and adolescents in this study reported consuming SSB, whereas the US and Australian counterparts reported 64 and $62 \%$, respectively. This study found the average SSB consumption level per consumer to be 398 ( $\mathrm{SE}$ 18.2) g/d, which is lower than the consumption of SSB among US children and adolescents $(631 \mathrm{~g} / \mathrm{d})$ and higher than that of Australian children and adolescents ( $248 \mathrm{~g} / \mathrm{d}$ ). The link between $\mathrm{Na}$ intake and SSB consumption and its impact on obesity should be explored further.

One can also propose that individuals with high $\mathrm{Na}$ intake would also practise bad health behaviours leading to adiposity that is, the association between $\mathrm{Na}$ intake and adiposity is an outcome of clustering of unhealthy behaviours ${ }^{(43)}$. This study controlled for physical activity and consumption of SSB and still found significant associations between $\mathrm{Na}$ intake and adiposity independent of physical activity. This finding indicates a possibility of an independent relationship between $\mathrm{Na}$ intake and adiposity.

Another possible mechanism between $\mathrm{Na}$ intake and obesity is that higher $\mathrm{Na}$ intake would result in higher water retention leading to higher body weight. This study used TBPF in an effort to exclude Na-related water retention as a possible reason for the association between $\mathrm{Na}$ intake and adiposity. This study found significant associations between urinary $\mathrm{Na}$ :creatinine ratio and TBPF among boys in a full model, whereas the association was relatively weaker among girls (model 2, Table 2). The association of TBPF with dietary Na intake was found to be weaker (Table 3). This possible mechanism appears to be worth further examination.

Previous studies have reported sex differences in the associations between $\mathrm{Na}$ intake and obesity. Song et al. ${ }^{(11)}$ analysed the data of adults from KNHANES 2007-2009 and 
Table 2. Risk of adiposity* by urinary sodium:creatinine ratio

(Odds ratios and $95 \%$ confidence intervals)

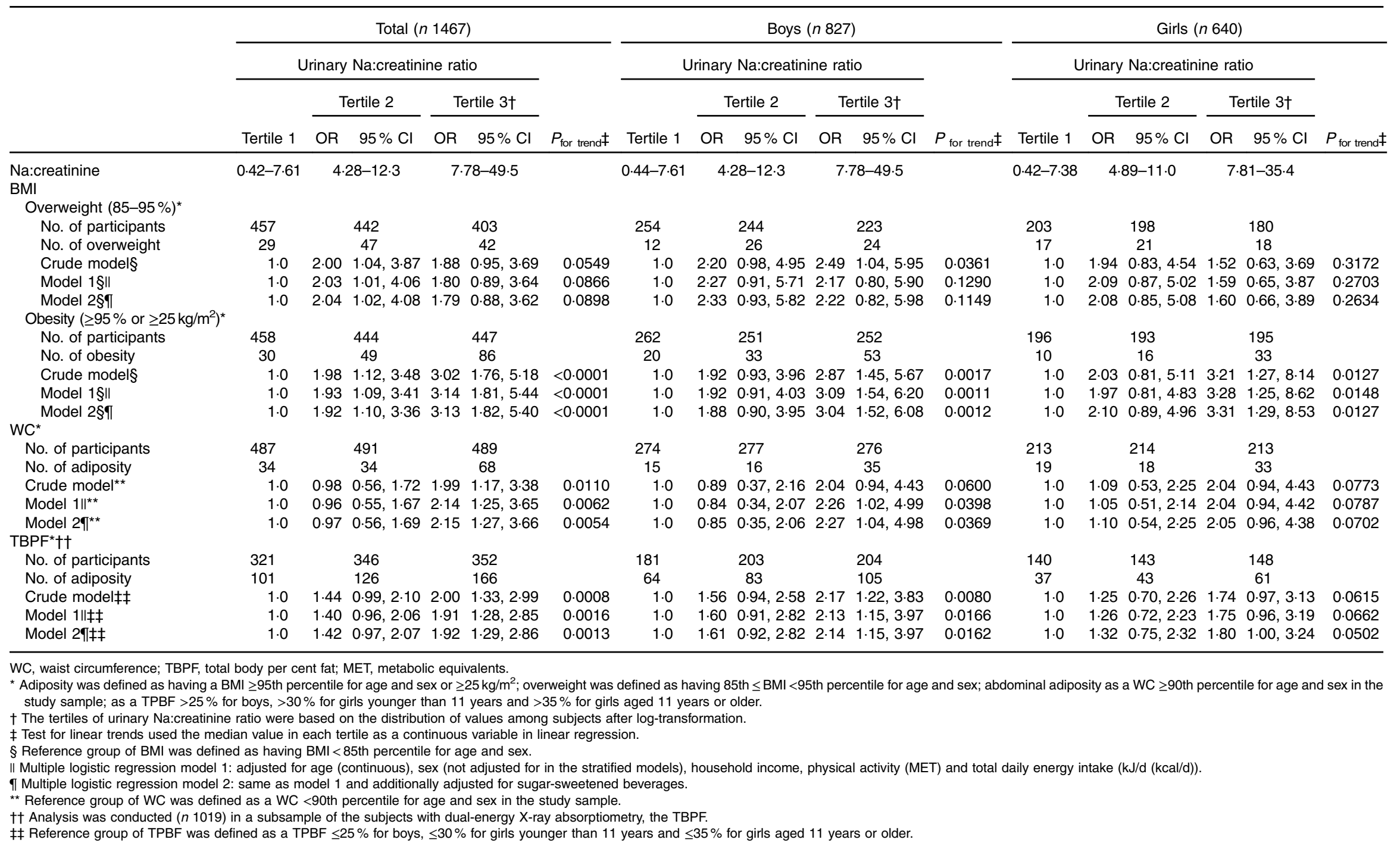


Table 3. Risk of adiposity* by dietary sodium intake

(Odds ratios and $95 \%$ confidence intervals)

\begin{tabular}{|c|c|c|c|c|c|c|c|c|c|c|c|c|c|c|c|c|c|c|}
\hline & \multicolumn{6}{|c|}{ Total $(n$ 1467) } & \multicolumn{6}{|c|}{ Boys ( $n$ 827) } & \multicolumn{6}{|c|}{ Girls $(n 640)$} \\
\hline & \multicolumn{5}{|c|}{ Dietary $\mathrm{Na}$ intake $(\mathrm{mg} / 4184 \mathrm{~kJ}(1000 \mathrm{kcal}))$} & \multirow[b]{3}{*}{$P_{\text {for trend }} \neq$} & \multicolumn{5}{|c|}{ Dietary Na intake (mg/4184 kJ (1000 kcal)) } & \multirow[b]{3}{*}{$P_{\text {for trend }} \neq$} & \multicolumn{5}{|c|}{ Dietary $\mathrm{Na}$ intake $(\mathrm{mg} / 4184 \mathrm{~kJ}(1000 \mathrm{kcal}))$} & \multirow[b]{3}{*}{$P_{\text {for trend } \ddagger}$} \\
\hline & \multirow[b]{2}{*}{ Tertile 1} & \multicolumn{2}{|c|}{ Tertile 2} & \multicolumn{2}{|c|}{ Tertile 3† } & & \multirow[b]{2}{*}{ Tertile 1} & \multicolumn{2}{|c|}{ Tertile 2} & \multicolumn{2}{|c|}{ Tertile 3† } & & \multirow[b]{2}{*}{ Tertile 1} & \multicolumn{2}{|r|}{ Tertile 2} & \multicolumn{2}{|c|}{ Tertile 3† } & \\
\hline & & OR & $95 \% \mathrm{Cl}$ & OR & $95 \% \mathrm{Cl}$ & & & OR & $95 \% \mathrm{Cl}$ & OR & $95 \% \mathrm{Cl}$ & & & OR & $95 \% \mathrm{Cl}$ & OR & $95 \% \mathrm{Cl}$ & \\
\hline $\mathrm{Na}$ (range) & $154-1589$ & & $146-2304$ & 204 & $6-12039$ & & $307-1589$ & & 457-2304 & & $51-6575$ & & $154-1492$ & & $446-2173$ & 204 & $16-12039$ & \\
\hline \\
\hline \multicolumn{19}{|l|}{ Overweight (85-95\%)* } \\
\hline No. of participants & 449 & & 443 & & 410 & & 249 & & 248 & & 224 & & 200 & & 195 & & 186 & \\
\hline No. of overweight & 29 & & 40 & & 49 & & 15 & & 24 & & 23 & & 14 & & 16 & & 26 & \\
\hline Crude model§ & 1.0 & 1.28 & $0 \cdot 75,2 \cdot 20$ & $2 \cdot 24$ & $1.24,4.04$ & 0.0091 & 1.0 & 1.26 & $0.61,2.58$ & 1.84 & $0.84,4.03$ & 0.1266 & 1.0 & 1.32 & $0.56,3.13$ & $2 \cdot 64$ & $1.19,5.86$ & 0.0184 \\
\hline Model $1 \S \|$ & 1.0 & 1.35 & $0.79,2 \cdot 32$ & $2 \cdot 11$ & $1.14,3.90$ & 0.0207 & 1.0 & 1.46 & $0.68,3.15$ & 1.68 & $0.71,3.98$ & 0.2351 & 1.0 & 1.33 & $0.56,3 \cdot 13$ & $2 \cdot 59$ & $1 \cdot 14,5 \cdot 84$ & 0.0202 \\
\hline Model 2§ף & $1 \cdot 0$ & 1.41 & $0.82,2 \cdot 41$ & $2 \cdot 23$ & $1.22,4.08$ & 0.0116 & 1.0 & 1.64 & $0.79,3.43$ & 1.91 & $0.81,4.50$ & 0.1414 & $1 \cdot 0$ & 1.33 & $0.58,3.07$ & $2 \cdot 60$ & $1.19,5.69$ & 0.014 \\
\hline \multicolumn{19}{|c|}{ Obesity $\left(\geq 95 \% \text { or } \geq 25 \mathrm{~kg} / \mathrm{m}^{2}\right)^{\star}$} \\
\hline No. of participants & 458 & & 451 & & 440 & & 259 & & 253 & & 253 & & 199 & & 198 & & 187 & \\
\hline No. of obesity & 38 & & 48 & & 79 & & 25 & & 29 & & 52 & & 13 & & 19 & & 27 & \\
\hline Crude model§ & 1.0 & 1.36 & $0.79,2 \cdot 33$ & $2 \cdot 75$ & $1.68,4.50$ & $<0.0001$ & 1.0 & $1 \cdot 20$ & $0.59,2.43$ & 2.75 & $1.47,5 \cdot 15$ & 0.0015 & 1.0 & 1.65 & $0.69,3.95$ & $2 \cdot 76$ & $1 \cdot 20,6 \cdot 33$ & 0.0216 \\
\hline Model $1 \S \|$ & 1.0 & 1.41 & $0.82,2.43$ & 2.73 & $1.65,4.51$ & $<0.0001$ & 1.0 & 1.29 & $0.63,2.65$ & $2 \cdot 71$ & $1 \cdot 42,5 \cdot 17$ & 0.0024 & 1.0 & 1.69 & $0.71,4.03$ & $2 \cdot 65$ & $1.17,5.98$ & 0.0277 \\
\hline Model 2§ণ & 1.0 & 1.44 & $0.83,2.49$ & $2 \cdot 79$ & $1.66,4.68$ & $<0.0001$ & 1.0 & $1 \cdot 26$ & $0.61,2.58$ & $2 \cdot 61$ & $1.39,4.91$ & 0.0027 & 1.0 & 1.88 & $0.73,4.80$ & 2.99 & $1 \cdot 23,7 \cdot 24$ & 0.0226 \\
\hline \multicolumn{19}{|c|}{ s } \\
\hline No. of participants & 487 & & 491 & & 489 & & 274 & & 277 & & 276 & & 213 & & 214 & & 213 & \\
\hline No. of adiposity & 36 & & 38 & & 62 & & 17 & & 18 & & 31 & & 19 & & 20 & & 31 & \\
\hline Crude model ${ }^{\star *}$ & 1.0 & 1.00 & $0.56,1.78$ & 1.93 & $1 \cdot 16,3 \cdot 20$ & 0.0092 & 1.0 & 0.82 & $0.37,1.82$ & 2.07 & $0.97,4.42$ & 0.0525 & $1 \cdot 0$ & $1 \cdot 17$ & $0.52,2.61$ & 1.79 & $0.88,3.66$ & 0.0959 \\
\hline Adjusted model $1 \|^{\star \star}$ & 1.0 & 1.04 & $0.58,1.86$ & 1.95 & $1 \cdot 17,3 \cdot 26$ & 0.0086 & 1.0 & 0.95 & $0.42,2 \cdot 15$ & $2 \cdot 10$ & $0.96,4.61$ & 0.0597 & 1.0 & 1.16 & $0.52,2.60$ & 1.77 & $0.86,3.65$ & $0 \cdot 1067$ \\
\hline Adjusted model $2 \boldsymbol{\Upsilon}^{\star \star}$ & 1.0 & $1 \cdot 12$ & $0.62,2.03$ & $2 \cdot 14$ & $1.25,3.67$ & 0.0044 & 1.0 & 0.99 & $0.43,2.25$ & $2 \cdot 22$ & $1.02,4.82$ & 0.0400 & 1.0 & 1.29 & $0.56,2.99$ & 1.98 & $0.93,4.25$ & 0.0645 \\
\hline \multicolumn{19}{|l|}{ TBPF* } \\
\hline No. of participants & 343 & & 343 & & 333 & & 198 & & 199 & & 191 & & 145 & & 144 & & 142 & \\
\hline No. of adiposity & 125 & & 128 & & 140 & & 81 & & 84 & & 87 & & 44 & & 44 & & 53 & \\
\hline 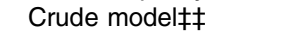 & $1 \cdot 0$ & 0.99 & $0.665,1.47$ & 1.32 & $0.91,1.90$ & 0.1401 & 1.0 & 0.99 & $0.61,1.63$ & 1.47 & $0.89,2.43$ & 0.1260 & $1 \cdot 0$ & 0.97 & $0.47,2.00$ & $1 \cdot 12$ & $0.60,2.09$ & 0.7149 \\
\hline Adjusted model $1 \| \ddagger \ddagger$ & 1.0 & 1.04 & $0.697,1.56$ & 1.31 & $0.89,1.92$ & 0.1735 & $1 \cdot 0$ & 1.01 & $0.604,1.69$ & 1.33 & $0 \cdot 76,2 \cdot 31$ & 0.3190 & $1 \cdot 0$ & 1.06 & $0.52,2 \cdot 18$ & $1 \cdot 18$ & $0 \cdot 65,2 \cdot 16$ & 0.5791 \\
\hline 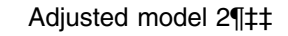 & 1.0 & 1.06 & $0.70,1.61$ & 1.33 & $0.89,1.99$ & 0.1577 & $1 \cdot 0$ & 1.02 & $0.61,1.71$ & 1.34 & $0.77,2.31$ & 0.9991 & $1 \cdot 0$ & 1.07 & $0.52,2.23$ & 1.23 & $0.66,2 \cdot 32$ & 0.0643 \\
\hline
\end{tabular}

WC, waist circumference; TBPF, total body per cent fat; MET, metabolic equivalents.

* Adiposity was defined as having a BMI $\geq 95$ th percentile for age and sex or $\geq 25 \mathrm{~kg} / \mathrm{m}^{2}$; overweight was defined as having 85 th $\leq \mathrm{BMI}<95$ th percentile for age and sex; abdominal adiposity as a WC $\geq 90$ th percentile for age and sex in the study sample; as a TPBF $>25 \%$ for boys, $>30 \%$ for girls younger than 11 years and $>35 \%$ for girls aged 11 years or older.

$\dagger$ The tertiles of dietary $\mathrm{Na}$ intake were based on the distribution of values among subjects after log-transformation

‡ Test for linear trend used the median value in each tertile as a continuous variable in linear regression.

$\S$ Reference group of $\mathrm{BMI}$ was defined as having $\mathrm{BMl}<85$ th percentile for age and sex.

II Multiple logistic regression model 1: adjusted for age (continuous), sex (not adjusted for in the stratified models), household income, total daily energy intake and physical activity (MET).

II Multiple logistic regression model 2: same as model 1 and additionally adjusted for sugar-sweetened beverages.

"Reference group of WC was defined as a WC <90th percentle for age and sex in the study sample.

列 
found significant associations between $\mathrm{Na}$ intake and obesity in men but not in women. Jain et al. ${ }^{(13)}$ found the opposite in the US population - that is, significant associations after controlling for covariates in women but not in men. This study found that different adiposity measures showed more apparent associations with $\mathrm{Na}$ intake in different sexes. Risk of overweight assessed by BMI was significantly associated with dietary $\mathrm{Na}$ intake only in girls, whereas adiposity risk assessed by WC was significantly associated with dietary $\mathrm{Na}$ intake only in boys. Risks of adiposity assessed by BMI and TBPF were in general consistent in both sexes. These inconsistent sex differences need to be further explored.

This study was limited by the cross-sectional nature of KNHANES; therefore, temporal relationship between $\mathrm{Na}$ intake and adiposity could not be established. Morning spot urine rather than 24-h urine samples were used in this study. Although the 24-h urine measurement is the gold standard for assessing salt intake through urinary $\mathrm{Na}$ excretion in individuals and in populations, it is often deemed inconvenient for repeated use in large population studies. There are concerns that a high participation burden, a lack of completeness and a high cost will affect the response rate and the practicality of using the test. Alternative methods such as spot and timed urine samples have been derived in an attempt to overcome this concern ${ }^{(33)}$. The validity of spot urine samples, however, remains controversial. A recent review showed that the correlations between mean $\mathrm{Na}$ amount from 24-h urine samples and that from overnight urine samples ranged from 0.62 to 0.95 among children and adolescents, although the authors concluded reliability of alternative methods to replace the 24-h urine $\mathrm{Na}$ test ${ }^{(44)}$. Although an equation has been developed to convert $\mathrm{Na}$ from spot urine samples to 24-h urine samples among adults ${ }^{(21)}$, no such equation exists for children. Therefore, this study utilised urinary $\mathrm{Na}$ :creatinine ratio value from spot urine samples without converting it to that of 24-h urine samples. Valid and reliable methods to replace 24-h urine $\mathrm{Na}$, including developing equations to convert $\mathrm{Na}$ from spot urine to 24-h $\mathrm{Na}$ in children, are much needed. Finally, adjustment with urinary $\mathrm{K}$ has been recommended in using urinary $\mathrm{Na}$ excretion ${ }^{(5)}$; however, this study was not able to do so because KNHANES did not test for urinary $\mathrm{K}$

One of the most meaningful contributions of this study was testing multiple assessment methods of not only $\mathrm{Na}$ intake but also adiposity. $\mathrm{Na}$ intake was assessed by dietary $\mathrm{Na}$ intake from 24-h recalls and urinary $\mathrm{Na}$ excretion, and significant associations with adiposity were found. For the outcome variable, adiposity, three different assessment methods (BMI, WC and TBPF) were used. This study found that associations with risk of obesity assessed by BMI were most consistent and stronger than other measures, whereas TBPF showed relatively weak association, showing no significant association with dietary $\mathrm{Na}$ intake. Using the DEXA method, one of the better assessment methods for total body fat, was a particular strength of this study. The significant findings regardless of assessment methods strongly support the associations between $\mathrm{Na}$ intake and adiposity, with suggestions for further studies on mechanisms of the association between $\mathrm{Na}$ intake and adiposity and needs of valid and easier assessment methods for $\mathrm{Na}$ intake among children.
In conclusion, this study demonstrated the significant associations between $\mathrm{Na}$ intake and adiposity in children and adolescents, independent of SSB consumption. The sex difference in this association warrants more research along with further investigations to establish mechanisms for the association. The findings of this study strongly support the worldwide campaign for $\mathrm{Na}$ intake reduction and suggest that the campaign would help decrease not only the traditionally discussed Na-related diseases such as hypertension, CVD and stroke but also adiposity.

\section{Acknowledgements}

The authors thank the members of the Korea Center for Disease Control who conducted the national survey.

This study was financially supported by an Inha University Research Grant (S.-K. L. 39208) and grants from the National Cancer Center in Korea (M. K. K. NCC-1310361).

The authors' contributions are as follows: M. K. K. designed and conducted the present study, arranged the data and performed the statistical analyses; S.-K. L. was involved in the interpretation of the results and drafted the paper. All the authors contributed to the development of the manuscript.

The authors declare that there are no conflicts of interest.

\section{References}

1. He FJ, Li J \& Macgregor GA (2013) Effect of longer-term modest salt reduction on blood pressure: Cochrane systematic review and meta-analysis of randomized trials. BMJ 346, f1325.

2. Vollmer WM, Sacks FM, Ard J, et al. (2001) Effects of diet and sodium intake on blood pressure: subgroup analysis of the DASH-sodium trial. Ann Intern Med 135, 1019-1028.

3. Nagata C, Takatsuka N, Shimizu N, et al. (2004) Sodium intake and risk of death from stroke in Japanese men and women. Stroke 35, 1543-1547.

4. Tuomilehto J, Jousilahti P, Rastenyte D, et al. (2001) Urinary sodium excretion and cardiovascular mortality in Finland: a prospective study. Lancet 357, 848-851.

5. Committee on the Consequences of Sodium Reduction in Populations (2013) Sodium Intake in Populations: Assessment of Evidence. Washington, DC: National Academy of Sciences.

6. Shikata K, Kiyohara Y, Kubo M, et al. (2006) A prospective study of dietary salt intake and gastric cancer incidence in a defined Japanese population: the Hisayama study. Int $J$ Cancer 119, 196-201.

7. Tsugane S \& Sasazuki S (2007) Diet and the risk of gastric cancer: review of epidemiological evidence. Gastric Cancer 10, 75-83.

8. $\mathrm{Hu} \mathrm{G}$, Jousilahti $\mathrm{P}$, Peltonen $\mathrm{M}$, et al. (2005) Urinary sodium and potassium excretion and the risk of type 2 diabetes: a prospective study in Finland. Diabetologia 48, $1477-1483$

9. Dahl LK, Silver L \& Christie RW (1958) The role of salt in the fall of blood pressure accompanying reduction in obesity. $N$ Engl J Med 258, 1186-1192.

10. Venezia A, Barba G, Russo O, et al. (2010) Dietary sodium intake in a sample of adult male population in southern Italy: results of the Olivetti Heart Study. Eur J Clin Nutr 64, 518-524.

11. Song HJ, Cho YG \& Lee HJ (2013) Dietary sodium intake and prevalence of overweight in adults. Metabolism 62, 703-708. 
12. Yoon YS \& Oh SW (2013) Sodium density and obesity: the Korea National Health and Nutrition Examination Survey 2007-2010. Eur J Clin Nutr 67, 141-146.

13. Jain N, Minhajuddin AT, Neeland IJ, et al. (2014) Association of urinary sodium-to-potassium ratio with obesity in a multiethnic cohort. Am J Clin Nutr 99, 992-998.

14. Larsen SC, Angquist L, Sorensen TI, et al. (2013) 24h urinary sodium excretion and subsequent change in weight, waist circumference and body composition. PLOS ONE 8, e69689.

15. Ellison RC, Sosenko JM, Harper GP, et al. (1980) Obesity, sodium intake, and blood pressure in adolescents. Hypertension 2, 78-82.

16. Libuda L, Kersting M \& Alexy U (2012) Consumption of dietary salt measured by urinary sodium excretion and its association with body weight status in healthy children and adolescents. Public Health Nutr 15, 433-441.

17. Woodruff SJ, Fryer K, Campbell T, et al. (2014) Associations among blood pressure, salt consumption and body weight status of students from south-western Ontario. Public Health Nutr 17, 1114-1119.

18. Zhu H, Pollock NK, Kotak I, et al. (2014) Dietary sodium, adiposity, and inflammation in healthy adolescents. Pediatrics 133, e635-e642.

19. Caggiula AW, Wing RR, Nowalk MP, et al. (1985) The measurement of sodium and potassium intake. Am J Clin Nutr $\mathbf{4 2}$, 391-398.

20. Willett W (2013) Nutritional Epidemiology, 3rd ed. New York, NY: Oxford University Press.

21. Kawasaki T, Itoh K, Uezono K, et al. (1993) A simple method for estimating $24 \mathrm{~h}$ urinary sodium and potassium excretion from second morning voiding urine specimen in adults. Clin Exp Pharmacol Physiol 20, 7-14.

22. Liu K, Dyer AR, Cooper RS, et al. (1979) Can overnight urine replace 24-hour urine collection to asses salt intake? Hypertension 1, 529-536.

23. Watson RL \& Langford HG (1970) Usefulness of overnight urines in population groups. Pilot studies of sodium, potassium, and calcium excretion. Am J Clin Nutr 23, 290-304

24. Prentice AM \& Jebb SA (2001) Beyond body mass index. Obes Rev 2, 141-147.

25. He FJ, Marrero NM \& MacGregor GA (2008) Salt intake is related to soft drink consumption in children and adolescents: a link to obesity? Hypertension 51, 629-634.

26. Grimes CA, Wright JD, Liu K, et al. (2013) Dietary sodium intake is associated with total fluid and sugar-sweetened beverage consumption in US children and adolescents aged 2-18 y: NHANES 2005-2008. Am J Clin Nutr 98, 189-196.

27. Grimes CA, Riddell LJ, Campbell KJ, et al. (2013) Dietary salt intake, sugar-sweetened beverage consumption, and obesity risk. Pediatrics 131, 14-21.

28. Korea Center for Disease Control and Prevention (2013) Korea Health Statistics 2012: Korea National Health and Nutrition Examination Survey (KHNAES v-3). Seoul: KCDC.

29. Powles J, Fahimi S, Micha R, et al. (2013) Global, regional and national sodium intakes in 1990 and 2010: a systematic analysis of $24 \mathrm{~h}$ urinary sodium excretion and dietary surveys worldwide. BMJ Open 3, e003733.

30. Korea Center for Disease Control and Prevention (2012) Analytic Guidelines of the Fifth Korea National Health and Nutrition Examination Survey 2011. Seoul: KCDC.

31. Korea Center for Disease Control and Prevention (2012) Korean National Health and Nutrition Examination Survey 2009-2011. Seoul: KCDC.

32. Kim YO, Kim MK, Lee SA, et al. (2009) A study testing the usefulness of a dish-based food-frequency questionnaire developed for epidemiological studies in Korea. Br J Nutr 101, 1218-1227.

33. Brown IJ, Tzoulaki I, Candeias V, et al. (2009) Salt intakes around the world: implications for public health. Int J Epidemiol 38, 791-813.

34. Krebs NF, Himes JH, Jacobson D, et al. (2007) Assessment of child and adolescent overweight and obesity. Pediatrics 120, Suppl. 4, S193-S228.

35. Korea Center for Disease Control and Prevention (KCDC) and The Korean Pediatric Society (2008) 2007 Korean National Growth Charts. Seoul: Korea Center for Disease Control and Prevention and The Korean Pediatric Society.

36. Moon JS, Lee SY, Nam CM, et al. (2008) 2007 Korean National Growth Charts: review of development process and an outlook. Korean J Pediatr 51, 1-25.

37. Taylor RW, Jones IE, Williams SM, et al. (2000) Evaluation of waist circumference, waist-to-hip ratio, and the conicity index as screening tools for high trunk fat mass, as measured by dual-energy X-ray absorptiometry, in children aged 3-19 y. Am I Clin Nutr 72, 490-495.

38. Barlow SE (2007) Expert committee recommendations regarding the prevention, assessment, and treatment of child and adolescent overweight and obesity: summary report. Pediatrics 120, Suppl. 4, S164-S192.

39. Seo JY, Cho YG, Kang JH, et al. (2013) New diagnostic criteria for obesity and overweight in Korean children and adolescents using 2007 Korean National Growth Charts. Obes Res Clin Pract 7, e182-e189.

40. Prior BM, Cureton KJ, Modlesky CM, et al. (1997) In vivo validation of whole body composition estimates from dual-energy X-ray absorptiometry. J Appl Physiol (1985) 83, 623-630.

41. Asayama K, Ozeki T, Sugihara S, et al. (2003) Criteria for medical intervention in obese children: a new definition of 'obesity disease' in Japanese children. Pediatr Int 45, 642-646.

42. US Department of Agriculture \& US Department of Health and Human Services (2010) Dietary Guidelines for Americans, 2010. Washington, DC: US Department of Agriculture and US Department of Health and Human Services.

43. Buck D \& Frosini F (2012) Clustering of Unhealthy Behaviors Over Time: Implications for Policy and Practice. London: The King's Fund.

44. Ji C, Sykes L, Paul C, et al. (2012) Systematic review of studies comparing 24-hour and spot urine collections for estimating population salt intake. Rev Panam Salud Publica 32, 307-315. 